\title{
Knockdown of NF-E2-related factor 2 inhibits the proliferation and growth of U251MG human glioma cells in a mouse xenograft model
}

\author{
XIANG-JUN JI ${ }^{1}$, SUI-HUA CHEN ${ }^{2}$, LIN ZHU ${ }^{1}$, HAO PAN ${ }^{1}$, YUAN ZHOU ${ }^{1}$, WEI LI ${ }^{1}$, WAN-CHUN YOU ${ }^{1}$, \\ CHAO-CHAO GAO ${ }^{1}$, JIAN-HONG ZHU ${ }^{1}$, KUAN JIANG ${ }^{1}$ and HAN-DONG WANG ${ }^{1}$ \\ Departments of ${ }^{1}$ Neurosurgery and ${ }^{2}$ Ophthalmology, Jinling Hospital, Nanjing University \\ School of Medicine, Nanjing, Jiangsu 210002, P.R. China
}

Received December 20, 2012; Accepted March 28, 2013

DOI: $10.3892 /$ or.2013.2476

\begin{abstract}
NF-E2-related factor 2 (Nrf2) is a pivotal transcription factor of cellular responses to oxidative stress and recent evidence suggests that Nrf2 plays an important role in cancer pathobiology. However, the underlying mechanism has yet to be elucidated, particularly in glioma. In the present study, we investigated the role of Nrf2 in the clinical prognosis, cell proliferation and tumor growth of human glioblastoma multiforme (GBM). We detected overexpression of Nrf2 protein levels in GBM compared to normal brain tissues. Notably, higher protein levels of Nrf2 were significantly associated with poorer overall survival and 1-year survival for GBM patients. Furthermore, we constructed the plasmid Si-Nrf2 and transduced it into U251MG cells to downregulate the expression of Nrf2 and established stable Nrf2 knockdown cells. The downregulation of Nrf2 suppressed cell proliferation in vitro and tumor growth in mouse xenograft models. We performed
\end{abstract}

Correspondence to: Professor Han-dong Wang, Department of Neurosugery, Jinling Hospital, Nanjing University School of Medicine, 305 East Zhongshan Road, Nanjing, Jiangsu 210002, P.R. China E-mail: hdwang_nj@yahoo.com.cn

Professor Sui-hua Chen, Department of Ophthalmology, Jinling Hospital, Nanjing University School of Medicine, 305 East Zhongshan Road, Nanjing, Jiangsu 210002, P.R. China

E-mail: chensuihua2002@yahoo.com.cn

Abbreviations: ARE, antioxidant response element; BrdU, bromodeoxyuridine; BSA, bovine serum albumin; kDa, kilodalton; ELISA, enzyme-linked immunosorbent assay; GBM, glioblastoma multiforme; HIF-1 $\alpha$, hypoxia-inducible factor-1 $\alpha$; HO-1, heme oxygenase-1; IRS, immunoreactivity scores; MVD, microvessel density; Nrf2, NF-E2-related factor 2; NQO-1, NAD(P)H:quinone oxidoreductase-1; PBS, phosphate-buffered saline; PCR, polymerase chain reaction; RT, reverse transcription

Key words: glioblastoma multiforme, NF-E2-related factor 2, proliferation, Ki-67, caspase-3, CD31 immunohistochemistry staining to detect the protein levels of Nrf2, Ki-67, caspase-3 and CD31 in the xenograft tumors and found that the expression levels of Nrf2 and Ki-67 were much lower in the Si-Nrf2 group compared to the Si-control group. In addition, the number of caspase-3-positive cells was significantly increased in the Si-Nrf2 group. By analysis of microvessel density (MVD) assessed by CD31, the MVD value in the Si-Nrf2 group decreased significantly compared to the Si-control group. These findings indicate that the knockdown of Nrf2 may suppress tumor growth by inhibiting cell proliferation, increasing cell apoptosis and inhibiting angiogenesis. These results highlight the potential of Nrf2 as a candidate molecular target to control GBM cell proliferation and tumor growth.

\section{Introduction}

Glioblastoma multiforme (GBM), a grade IV and malignant glioma, is the most common, aggressive and proliferative among all gliomas (1). Due to its nature, and following high recurrence rate, as well as strong resistance to treatment, the prognosis of GBM patients remains poor even after surgical resection and adjuvant radiotherapy or chemotherapy. In 2001, Sullivan reported that the median survival time for GBM patients was approximately 12 months, compared with 5 years in patients with low-grade glioma (2). Despite decades of concerted efforts and the advances in surgery, radiotherapy and chemotherapy, the overall 5-year survival rate of GBM remains less than 5\% and is even poorer for elderly patients (3). Therefore, it is important to elucidate the mechanism of glioma tumorigenesis and to find and develop the key molecular targets for effective therapy.

NF-E2-related factor 2 (Nrf2) is considered a critical regulator of intracellular antioxidants and phase II detoxification enzymes. Nrf2 belongs to the Keap1-Nrf2-ARE (antioxidant response element) signaling pathway and upregulates several ARE-containing genes, including heme oxygenase-1 (HO-1) and NAD(P)H:quinone oxidoreductase-1 (NQO-1) (4). Nrf2 was formerly considered to help protect cells in normal tissues from harmful stimulus, including inflammation, trauma, ischemia, hemorrhage and cancer (5-9). However, recent findings 
suggest that Nrf 2 might play the dark role in tumors. A number of studies showed constitutively high levels of Nrf2 promote cancer formation and contribute to chemoresistance (4,10-12). Further investigation demonstrated that Nrf2 plays a pivotal role in cell proliferation by activating its target genes, including various detoxification enzymes, glutathione-related enzymes and cell-cycle regulatory proteins (13-15). Kim et al (16) showed that $\mathrm{Nrf} 2$ as a candidate molecular target might control colon tumor angiogenesis by imposing a blockade to HIF-1 $\alpha$ (hypoxia-inducible factor- $1 \alpha$ ) signaling. Recently, we also demonstrated that Nrf2 was involved in migration and invasion of U251MG glioma cells (17). Collectively, these results demonstrated that Nrf2 contributes to proliferation, migration, invasion, angiogenesis and chemoresistance observed in several types of malignant tumors. However, there are few reports on the function of Nrf2 in glioma. In the present pilot study, we further investigated the role of Nrf2 in the proliferation and tumor growth of human U251MG glioma cells in the nude mouse xenograft model following Nrf2 knockdown.

\section{Materials and methods}

Patients and tissue samples. The present study was approved by the Research Ethics Committee of Jinling Hospital, School of Medicine, Nanjing University, China. Written informed consent was obtained from all patients. Surgically removed human GBM tissue samples were continuously obtained paraffin-embedded from 49 patients (34 males and 15 females (2.23:1), average age 52 years (27-82) attended at Jinling Hospital from 2008 to 2010, according to the ethical and legal standards. All the slides were re-evaluated according to WHO classifications by two pathologists, with differences resolved by careful discussion (18). None of the patients had received chemotherapy or radiotherapy prior to the surgery. Human normal brain tissues (mostly from the cortex) were obtained from 5 male and 5 female patients in the pathway during surgical removal of deep benign tumor from 2010 to 2011 .

A total of 37 patients completed the follow-up until mortality and the survival time was censored in August 2012, while 12 patients dropped out of the study. No patients succumbed to a disease not directly related to their gliomas or due to unexpected events. Follow-up was conducted every 6 months by telephone. The adjuvant chemotherapy and radiotherapy were fully discussed with the patients prior to the treatment and all patients received the standard chemotherapy and radiotherapy. Overall survival (OS) was defined as the time from the date of surgery to the date of death from all cases. Progression-free survival (PFS) was defined as the time from surgery to the time of tumor progression on MRI, or mortality due to GB.

Cell culture, plasmid transfection and establishment of Nrf2 knockdown cells. Human U251MG glioma cells were obtained from the American Type Culture Collection (ATCC, Manassas, VA, USA). The cells were maintained in Dulbecco's modified Eagle's medium (Gibco, Los Angeles, CA, USA) supplemented with $10 \%$ fetal bovine serum (Gibco) and $1 \%$ penicillin/streptomycin (Gibco), and were incubated at $37^{\circ} \mathrm{C}$ in a $5 \% \mathrm{CO}_{2}$ incubator. The lentiviral particles with Nrf2 short hairpin RNA (shRNA) were purchased from GenePharma (Shanghai, China) and the target sequence was GCAGTTCAATGAAGCTCAACT. The new plasmid was termed Si-Nrf2. Random sequence, TTCTCCGAACGTGTCACGT, was used as the negative control, and was termed Si-control. Cell transfection was performed in 6-well plates with lentiviral particles containing either Si-control RNA or Nrf2 shRNA expression plasmid using Polybrene (GenePharma) according to the instructions of the manufacturer. Transfection was continued for $24 \mathrm{~h}$ and followed by a 24 -h recovery in the complete medium. For the selection of cells with target plasmids, cells were grown in the medium containing $1.5 \mu \mathrm{g} / \mathrm{ml}$ puromycin (Sigma-Aldrich, St. Louis, MO, USA) for up to 2 weeks. Then, the positive clonal cells were selected and cultured in 96-well plates with the medium containing $1.0 \mu \mathrm{g} / \mathrm{ml}$ puromycin.

Total RNA extraction and RT-PCR analysis. Total RNA was isolated from cells using the TRIzol reagent (Invitrogen) following the manufacturer's recommendations and subjected to DNase (Promega, Madison, WI, USA) treatment. Reverse transcription (RT) reaction was performed by incubating $400 \mathrm{ng}$ of total RNA with the first-strand cDNA synthesis kit (Takara, Dalian, China) following the manufacturer's recommendations. The concentration and purity of total RNA were determined by spectrophotometer analysis (OD260/280:1.82.2) and agarose gel electrophoresis. Obtained cDNA was amplified immediately using the following primers: for $\mathrm{Nrf} 2$, 5'-TCAGCGACGGAAAGAGTATGA-3' and 5'-CCACTGG TTTCTGACTGGATGT-3'; for NQO-1, 5'-ATGGTCGGC AGAAGAGC-3' and 5'-GGAAATGATGGGATTGAAGT-3'; for HO-1, 5'-TCTCCGATGGGTCCTTACACTC-3' and 5'-GGCATAAAGCCCTACAGCAACT-3'; and for GAPDH, 5'-GAAATCCCATCACCATCTTC-3' and 5'-GGACTCCAC GACGTACTCA-3'. The amplification and data acquisition were carried out on a real-time PCR system (Agilent, Anaheim, CA, USA) using FastStart Universal SYBR Green Master (Roche, Mannheim, Germany). The conditions were predenaturated at $95^{\circ} \mathrm{C}$ for $10 \mathrm{~min}$, followed by 40 cycles at $95^{\circ} \mathrm{C}$ for $15 \mathrm{sec}$ and $60^{\circ} \mathrm{C}$ for $1 \mathrm{~min}$. All samples were analyzed in triplicates in three independent experiments. Reaction without cDNA was used as no-template control, and no-RT controls were also set up to rule out genomic DNA contamination. Relative quantification of mRNA expression was determined using the $2^{-\Delta \Delta C q}$ method. PCR amplification efficiency of each gene was established by means of calibration curves. All the real-time PCR experiments were performed in accordance to the minimum information for publication of quantitative realtime PCR experiments (19).

Western blot analysis. Total protein was separated by $8 \%$ SDS-polyacrylamide gel electrophoresis using the Criterion system (Bio-Rad Laboratories, Hercules, CA, USA) at a constant voltage of $90 \mathrm{~V}$. Proteins were subsequently transferred to PVDF membranes (Millipore, Billerica, MA, USA) at a constant voltage of $15 \mathrm{~V}$ for $30 \mathrm{~min}$. Non-specific binding sites were blocked with $3 \%$ skim milk for $2 \mathrm{~h}$ at room temperature and incubated overnight with primary antibodies at $4^{\circ} \mathrm{C}$. The following antibodies were used: 1:500 anti-Nrf2 (Abcam, Cambridge, MA, USA), 1:1,000 anti-HO-1 (Abcam), 1:2,000 anti- $\beta$-actin (Santa Cruz Biotechnology, Santa Cruz, 
CA, USA). The membranes were then incubated with the appropriate secondary antibodies (Cell Signaling Technology, Danvers, MA, USA; 1:2,000) for $2 \mathrm{~h}$ at room temperature. After washing, protein bands were visualized with Chemiluminescent HRP Substrate (Millipore) for $5 \mathrm{~min}$ at room temperature and exposed to X-ray film (Fuji Hyperfilm). Relative changes in protein expression were estimated from the mean pixel density using Quantity One software 4.6.2 (Bio-Rad Laboratories), normalized to $\beta$-actin and presented as relative density units.

Cell proliferation assay. Cell proliferation was measured by Cell-Counting kit-8 (CCK-8) (Dojindo, Kumamoto, Japan) and cell proliferation ELISA kit (Roche) according to the manufacturer's instruction. Cells were plated in 96-well plates at a density of $2 \times 10^{3}$ cells/well. After the incubation for 24 and $48 \mathrm{~h}, 10 \mu \mathrm{l} \mathrm{CCK}-8$ were added and cells were further incubated for $1 \mathrm{~h}$. The absorbance was then measured at $450 \mathrm{~nm}$ using an enzyme-linked immunosorbent assay (ELISA) microplate reader (Bio-Rad Laboratories). DNA synthesis of cells was measured using the ELISA kit, which is a colorimetric immunoassay based on the assessment of bromodeoxyuridine (BrdU) incorporation during DNA synthesis. Cells were plated in 96 -well plates at a density of $2 \times 10^{3}$ cells/well and grown for $24 \mathrm{~h}$. Then, cells were labeled with $10 \mu \mathrm{M}$ BrdU for $2 \mathrm{~h}$ and incorporated BrdU was quantified using a plate reader at $492 \mathrm{~nm}$ using the ELISA microplate reader (Bio-Rad Laboratories).

Tumor xenograft study. Animal studies were performed according to the institutional Committee for Animal Research and in conformity with national guidelines for the care and use of laboratory animals. Si-Nrf2 and Si-control-stabletransfected cells and U251MG cells $\left(5.0 \times 10^{6}\right)$ were suspended in $100 \mu 1$ phosphate-buffered saline (PBS) and then injected subcutaneously into either side of the posterior flank of the male BALB/c athymic nude mice (Charles River Breeding Laboratories, Wilmington, MA, USA) at 5-6 weeks of age. Tumor growth was examined weekly for at least 5 weeks. Tumors were then stripped and weighed before paraffinembedding. Tumor volumes were determined by external measurements and calculated according to $\mathrm{V}=\left[\mathrm{L} \mathrm{x} \mathrm{W}^{2}\right] \times 0.52$, where $\mathrm{V}$ is the volume, $\mathrm{L}$ is the length and $\mathrm{W}$ is the width.

Immunohistochemical staining. For the immunohistochemical analysis of human brain tissues and xenograft tumors, 3- $\mu \mathrm{m}$ serial sections were dewaxed, and endogenous peroxidase was quenched with $3 \% \mathrm{H}_{2} \mathrm{O}_{2}$ in methanol for $30 \mathrm{~min}$ (20). Before staining, non-specific binding was blocked by incubation with $10 \%$ bovine serum albumin (BSA) in PBS at $37^{\circ} \mathrm{C}$ for $1 \mathrm{~h}$. Then, all incubations with 1:50 anti-Nrf2 (Abcam), 1:200 anti-Ki-67 (Abcam), 1:50 anti-caspase-3 (Abcam) and 1:100 anti-CD31 (Abcam) antibodies in PBS containing 1\% BSA were carried out at $4{ }^{\circ} \mathrm{C}$ overnight. All sections were briefly washed in PBS and incubated at room temperature with the anti-rabbit antibody and avidin-biotin peroxidase (Vector Laboratories Inc., Burlingame, CA, USA). Color was then developed by incubation with the diaminobenzidine solution (Dako Corp., Carpinteria, CA, USA). Nuclei were counterstained blue with Meyer's hematoxylin (Sigma-Aldrich). Non-neoplastic brain tissues were used as control and non-immune $\operatorname{IgG}$ was used as negative control antibody for the staining. The slides were scored by two independent observers. The number of positivestaining cells was counted from two areas of each specimen and the percentage of positive cells was calculated. The staining intensity was estimated and stratified as 0 (negative), 1 (weak), 2 (moderate) and 3 (strong). The immunohistochemistry positive results for Nrf2 were recorded as previously described (20). Then we scored the percentage of immunoreactive tumor cells as $0(0 \%), 1(1-10 \%), 2(11-50 \%)$ and 3 $(>50 \%)$. Final immunoreactivity scores (IRS) were obtained for each specimen by multiplying the percentage and the intensity score. We then estimated the protein expression levels by classifying IRS values as low (based on an IRS value $<5$ ) and as high (based on an IRS value $>5$ ).

Microvessel density. Microvessel density (MVD) of the tumor tissues was assessed through the endothelial marker CD31 immunohistochemical analysis and determined according to the method previously described (21). The immunostained sections were initially screened at low magnification (x50) to identify hot spots of the neovascularization. Any yellow-brown stained endothelial cell or endothelial cell cluster clearly separate from the adjacent microvessels, tumor cells and other connective tissue elements was considered a single, countable microvessel. Within the hot spot area, the stained microvessels were counted in a single high-power (x200) field, and the average vessel count in three hot spots was considered the value of MVD. All counts were performed by three investigators in a blinded manner. Microvessel counts were compared between the observers and discrepant results were reassessed. The consensus was used as the final score for analysis (22).

Statistical analysis. SPSS 19.0 software was used for statistical analysis. OS was reported in months and defined as the interval between the date of the surgery and the date of death. OS curves were estimated by the Kaplan-Meier method, and the difference in survival was evaluated using the log-rank test. Statistical data are presented as the means \pm SD. One-way analysis of variance (ANOVA) followed by Tukey post-hoc comparison tests was used to compare the levels of different experimental groups. P-values of $<0.05$ and $<0.01$ were considered to indicate statistically significant differences.

\section{Results}

Overexpression of Nrf2 in human GBM tissues and the higher expression levels of Nrf2 correlate with shorter survival. Nrf2 protein expression levels were investigated by immunohistochemical analysis in 49 paraffin-embedded GBM samples, 15 adjacent normal tissues and 10 normal tissues. Immunoreactivity for the Nrf2 antigen was seen in 43/49 $(87.78 \%)$ of the tumor samples and in $1 / 15(6.67 \%)$ adjacent normal tissues, and the 10 normal brain tissues were negative for Nrf2 (Fig. 1). The Nrf2-positive label was confined mainly to the cytoplasm, with some positive labels in the nuclei. Of the 37 tumor samples for the follow-up patients, high expression levels of Nrf2 were detected in $40.5 \%$ (15/37) and $59.5 \%$ (22/37) of patients, respectively. The log-rank test showed that the expression levels of $\mathrm{Nrf} 2$ were significantly correlated with 

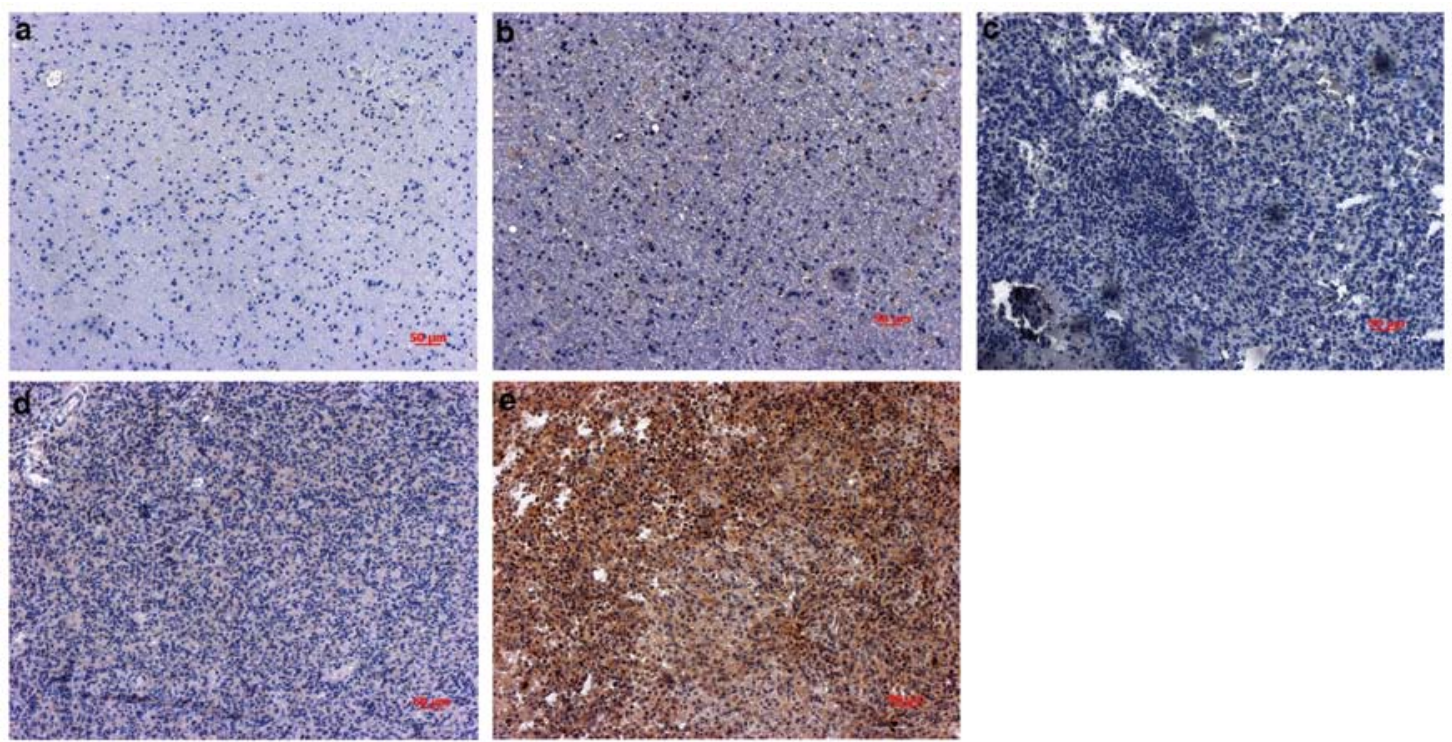

Figure 1. Overexpression of Nrf2 in immunohistochemical analysis. (a) Normal tissues and (b) adjacent normal tissues were negative for Nrf2. (c) Six GBM tissues were negative and 43 tissues were positive for Nrf2, including (d) low protein levels and (E) high protein levels. Bar, $50 \mu \mathrm{m}$.
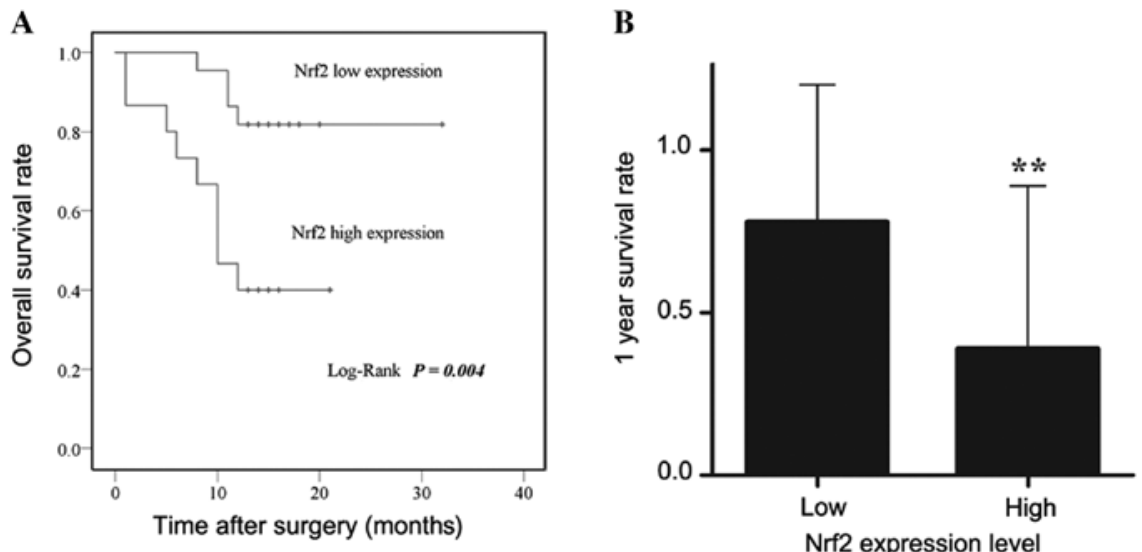

Figure 2. Clinical significance of Nrf2 expression in GBM. (A) The log-rank test showed the protein levels of Nrf2 were significantly correlated with the overall survival of GBM patients $(\mathrm{P}=0.004)$. (B) One-year survival rates for GBM patients based on the immunoreactivity of Nrf2 $(\mathrm{P}<0.01) .{ }^{* *} \mathrm{P}<0.01$.

the OS of GBM patients ( $\mathrm{P}=0.004$, Fig. 2A). Thus, patients with high expression levels of Nrf2 (IRS value $>5$ ) had shorter OS than the patients with low expression levels (IRS value $<5$ ) $(28.09 \pm 1.77$ vs. $12.60 \pm 1.93$ months, respectively). We further evaluated whether the Nrf2 immunoreactivity was correlated with 1-year survival rates, and found the 1-year survival rates to be $40.0 \%$ (18/22) for patients with high Nrf2 expression and $81.8 \%$ (6/15) for patients with low Nrf2 expression ( $\mathrm{P}=0.0079$, Fig. 2B). These results highlight the clinical significance of Nrf2 in determining the prognosis for patients with GBM.

Stable transfection effect on the $m R N A$ and protein levels of Nrf2. To further investigate the role of Nrf2 in glioma cell proliferation and tumor growth, we established an Nrf2 knockdown U251MG cell line. We transduced cells with plasmids encoding non-specific Si-control RNA and Nrf2targeting shRNA with lentiviral particles. The stable cell line was obtained following puromycin selection. Compared to the Si-control, cells with stable Si-Nrf2 expression showed repressed transcript levels of Nrf2 and its target genes, such as the catalytic subunit of HO-1 and NQO-1 (Fig. 3A-C). Similar patterns were measured by Nrf2 and HO-1 immunoblot analysis. The total protein levels were much lower in Si-Nrf2 cells in comparison to the Si-control (Fig. 4). In total, stable Si-Nrf2 transfection reduced the Nrf2 mRNA and protein level to 27 and $42 \%$ in comparison to the $\mathrm{Si}$-control, respectively $(\mathrm{P}<0.01)$.

Suppressed cell proliferation in vitro and tumor growth in Nrf2 knockdown xenograft. To investigate the role of Nrf2 in in vitro proliferation, we performed the CCK-8 assay and the BrdU incorporation assessment. First, we performed the CCK-8 assay at 24 and $48 \mathrm{~h}$ and found that the Si-Nrf2 cells were suppressed in cell proliferation rate, particularly at $48 \mathrm{~h}$ (Fig. 5A-C). Similarly, the assessment of BrdU incorporation confirmed the CCK-8 result (Fig. 5D). To further examine the role of Nrf2 in tumorigenesis, we implanted the stable transfected U251MG cells into nude mice and assessed the tumor growth. We measured the volume changes during tumor 

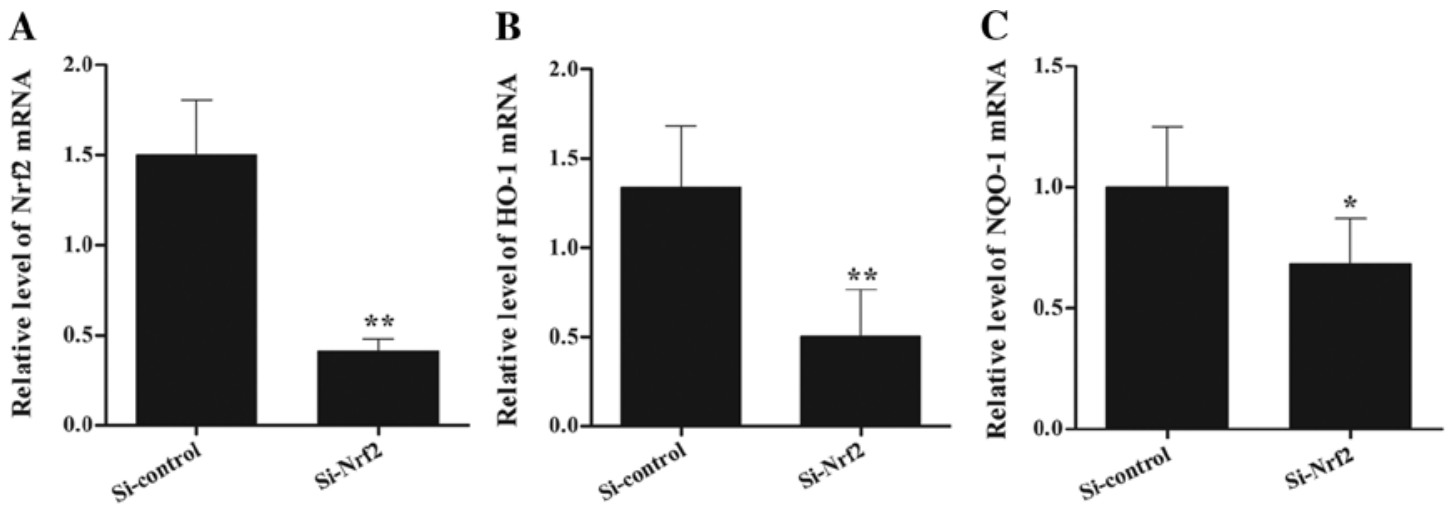

Figure 3. The mRNA levels of the control, the Si-control and the Si-Nrf2 group following stable transfection measured by real-time quantitative PCR. (A) The relative mRNA level of Nrf2 was decreased by $60 \%$ in the Si-Nrf2 group $(\mathrm{P}<0.01)$, with respect to the Si-control group. The decreased mRNA levels of HO-1 ( $\mathrm{B}, \mathrm{P}<0.01)$ and NQO-1 (C, $\mathrm{P}<0.05)$ confirmed the knockdown effects. Data are presented as the means $\pm \mathrm{SD}$; ${ }^{*} \mathrm{P}<0.05$, ${ }^{* *} \mathrm{P}<0.01$ vs. the control and the Si-control group.
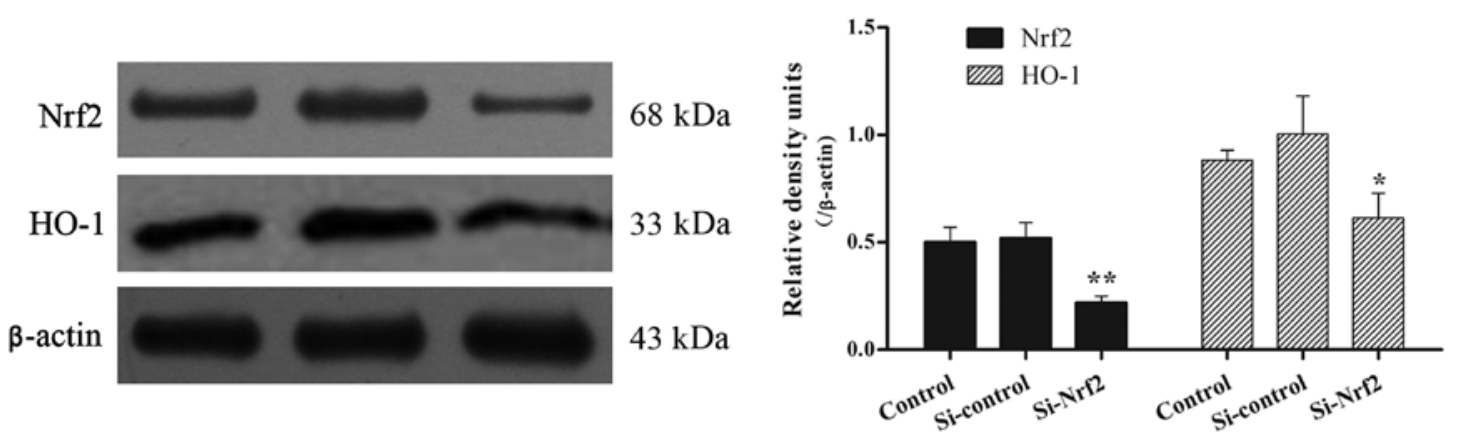

Figure 4. Stable transfection effect on the protein levels. The figure on the left shows the protein expression of Nrf2 and HO-1 using $\beta$-actin as the loading control, respectively in the control, the Si-control and the Si-Nrf2 group. The figure on the right shows the ratios of Nrf2/ $\beta$-actin and HO- $1 / \beta$-actin. Bar represents the means $\pm \mathrm{SD} ;{ }^{*} \mathrm{P}<0.05,{ }^{* *} \mathrm{P}<0.01$ vs. the control and the Si-control group. kDa, kilodalton.
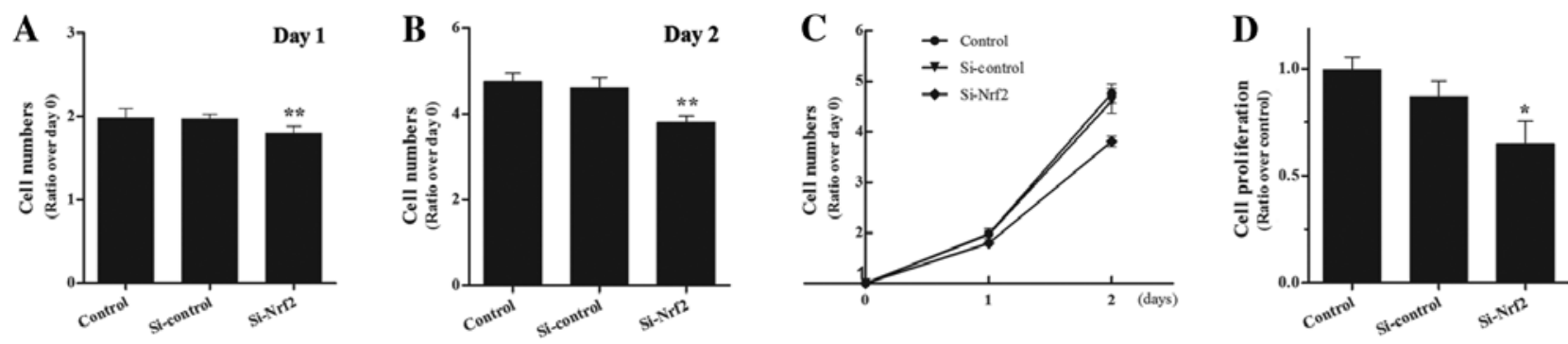

Figure 5. Suppressed U251MG cell proliferation in vitro. CCK-8 assay (A-C) was performed at 24 and $48 \mathrm{~h}$. The proliferation rate of the Si-Nrf2 group was suppressed at (A) $24 \mathrm{~h}$ and was significantly suppressed at (B) $48 \mathrm{~h}$. (C) The drawing lines show proliferation rates of three groups for $48 \mathrm{~h}$. (D) BrdU incorporation assessment by cell proliferation ELISA kit focused on the DNA synthesis of cells and confirmed the results of the CCK-8 assay. Bar represents the means $\pm \mathrm{SD} ;{ }^{*} \mathrm{P}<0.05,{ }^{* *} \mathrm{P}<0.01$ vs. the control and the Si-control group.

growth and the tumor weight in the end, and observed that tumor size of Si-Nrf2 cells increased more slowly than the size of Si-control cells (Fig. 6A). A similar result was observed in the tumor weight (Fig. 6B and C). Thus, the tumor growth of the Si-Nrf2 cells was markedly suppressed in comparison to the Si-control in the xenograft model.

Analysis of the immunohistochemical staining in the xenograft tumors. We performed immunohistochemistry analysis to detect the protein levels of Nrf2, Ki-67, caspase-3 and CD31 in the xenograft tumors (Figs. 7 and 8). Nrf2 was mainly detected in the cytoplasm with various expression levels and a few in the nuclei. Immunoreactivities in the tumor sections of Si-Nrf2 cells ranged from undetectable to low, whereas the immunoreactivities of Si-control cells were much higher (Fig. 7a-c and Fig. 8A). Ki-67, located in the nucleus of eukaryocytes, is a sensitive biological marker for cell proliferation (23). The expression level of Ki-67 in the sections of Si-Nrf2 cells was lower in comparison to the Si-control group (Fig. 7d-f and Fig. 8B). This observation confirmed the results 
A

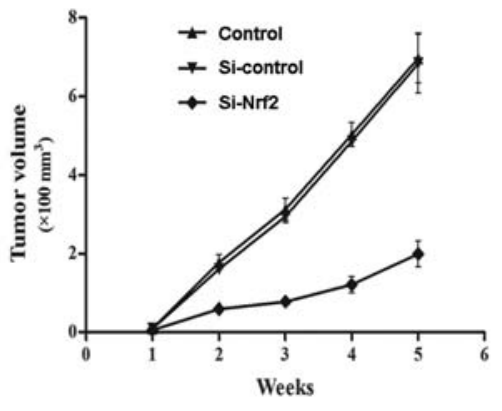

B

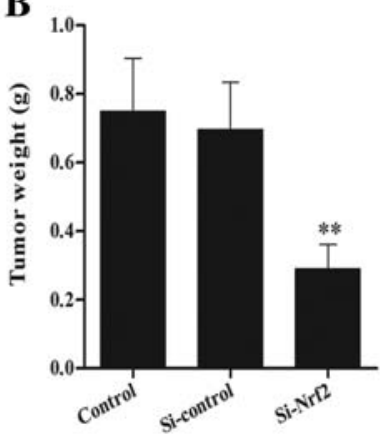

C

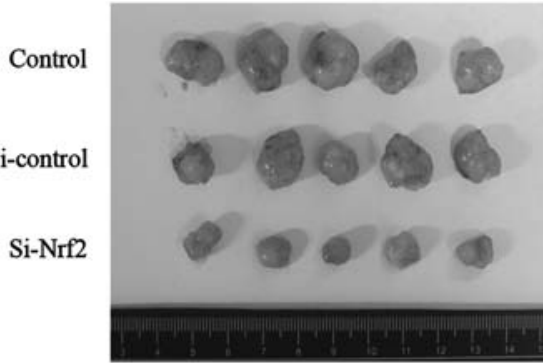

Figure 6. Tumor growth in Nrf2 knockdown xenografts. (A) Tumor volume of the Si-Nrf2 group increased slowly compared to the Si-control group. (B) Graphic presentation and (C) comparison of tumor weights. Bar represents the means $\pm \mathrm{SD} ;{ }^{* *} \mathrm{P}<0.01$ vs. the control and the Si-control group.

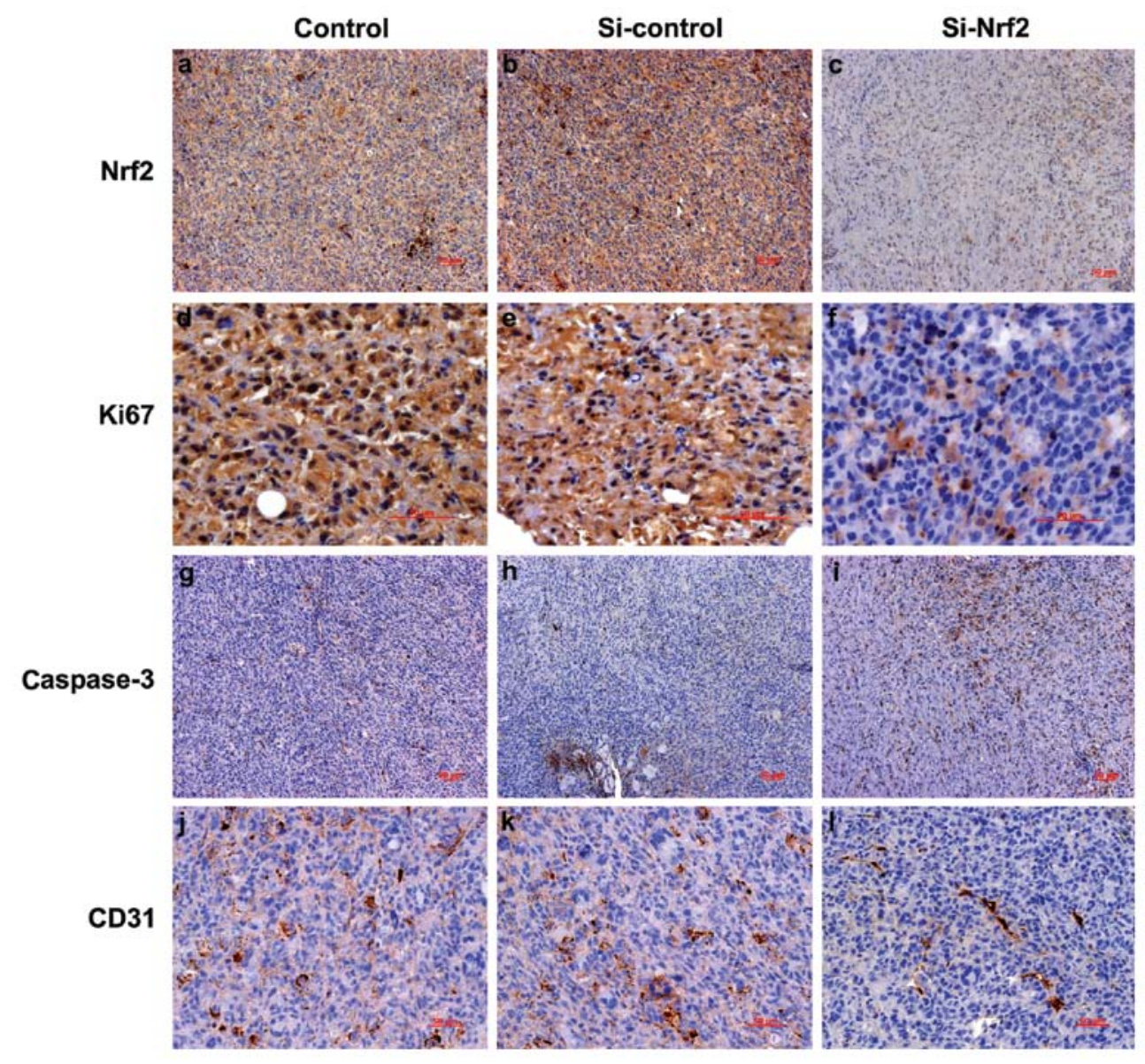

Figure 7. Representative images of immunohistochemical stainings in xenograft tumors. (a-c) Protein levels of Nrf2. Bar, $50 \mu$ m. (d-f) Protein levels of Ki-67 mainly detected in the nuclei. Bar, $50 \mu \mathrm{m}$. (g-i) Caspase-3 positive cells significantly increased in Nrf2-inhibited tumors. Bar, $50 \mu \mathrm{m}$. (J-L) Positive staining of endothelial marker CD31 detected in the endothelial membrane. Bar, $50 \mu \mathrm{m}$.

of cell proliferation assay in vitro. In order to further explore the mechanism of the tumor suppression, we detected the cell apoptosis and angiogenesis in the xenograft model. Coincident with reduced tumor mass of the Si-Nrf2 group, the number of caspase-3 positive cells was significantly increased in Nrf2-inhibited tumors (Fig. 7g-i and Fig. 8C). Subsequently, we performed immunohistochemical analysis of endothelial marker CD31 to assess the MVD in the tumor tissues. Compared with the Si-control group, MVD values in the Si-Nrf2 group decreased significantly (Fig. 7j-1 and Fig. 8D).

\section{Discussion}

The present study focused on human GBM, which is an aggressive tumor with heterogeneous tumor biology, high invasiveness, rapid tumor cell proliferation and poor prognosis. The results of our analysis showed that the protein level of Nrf2 in human GBM tissues was markedly upregulated in comparison to non-neoplastic brain tissues, and GBM patients with high Nrf2 expression tended to have poorer OS. Furthermore, stable downregulation of Nrf2 in GBM U251MG 

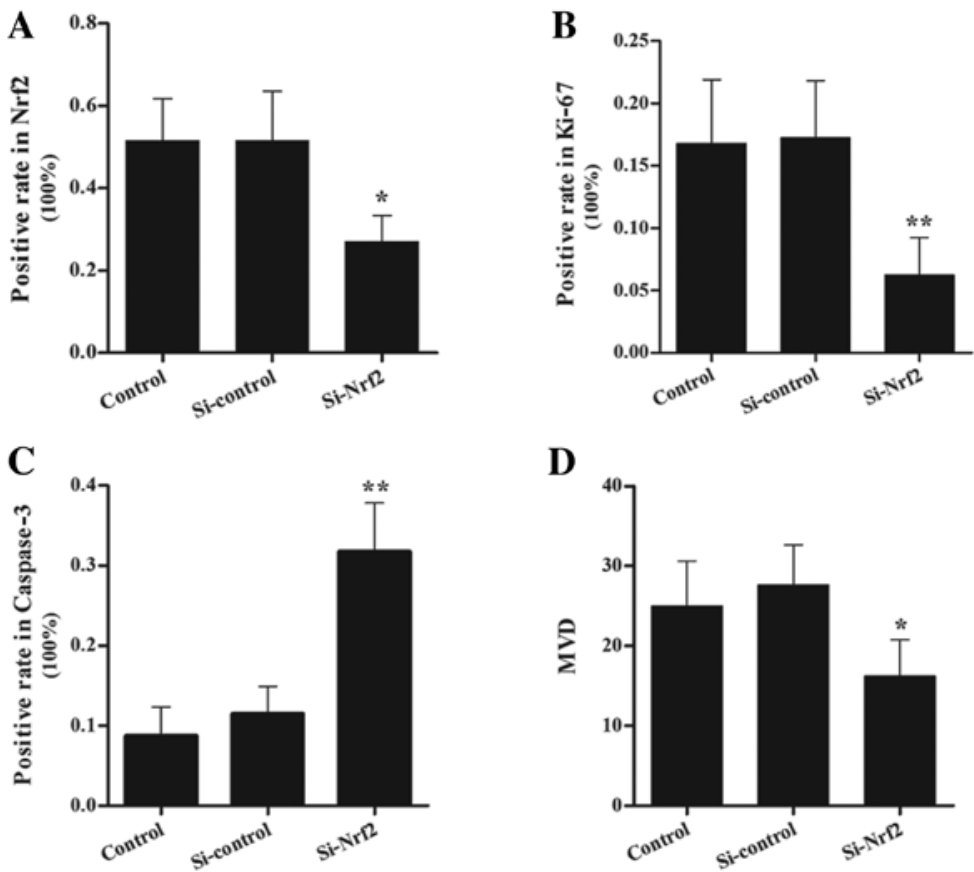

Figure 8. Immunohistochemical analysis of Nrf2, Ki-67, caspase-3 and CD31. (A) Note that immunoreactivity of Nrf2 was significantly suppressed in the Si-Nrf2 group. (B and C) Graphic representations of the positive staining percentage of Ki-67 and caspase-3. (D) MVD values assessed by CD31 staining. Bar represents the means $\pm \mathrm{SD} ;{ }^{*} \mathrm{P}<0.05,{ }^{* *} \mathrm{P}<0.01$ vs. the control and the $\mathrm{Si}-$ control group.

suppressed the cell proliferation in vitro and the tumor growth in a nude mice xenograft model. These findings suggest that Nrf2 is a valuable transcription factor in cell proliferation, tumor growth and clinical prognosis.

Although the cytoprotective roles of Nrf2 have been confirmed by extensive studies since the first report by Moi et al in 1994 (24), there are increasing concerns regarding the deleterious effects of the Nrf2 signal in cancer cell biology. Research on Nrf2 in cancer have been promoted by reports that Nrf2 is often overactivated in various cancer cells. Studies revealed that mutations of the Keap1 protein were identified from lung cancer cell lines and the tumor tissues of lung cancer patients $(25,26)$. These mutations caused the loss of Keap1 repression of $\mathrm{Nrf} 2$, and then led to the constitutive Nrf2 activation. Aside from Keap1, mutations of Nrf2 were also identified, and, due to their mutations, Nrf2 was overexpressed in other types of tumor tissues and cell lines (27-30). Merikallio et al (31) found that Nrf2 and its stabilizing protein DJ1 affected the prognosis of patients with lung cancer. Further studies indicated that $\mathrm{Nrf} 2$ played a pivotal role in tumor cell proliferation and chemoresistance $(13,15)$. These findings led to the hypothesis that inhibition of Nrf2 expression could reverse the phenotypic characteristics of cancer cells, such as rapid proliferation, unresponsiveness to apoptosis, drug resistance and angiogenesis. Studies have demonstrated that RNAi-mediated inhibition of Nrf2 expression in lung cancer cells could induce generation of reactive oxygen species, attenuate tumor growth, and circumvent chemoresistance in vitro and in vivo $(13,14,32)$. Our study focused on glioma and the results suggested that $\mathrm{Nrf} 2$ is involved in the cell proliferation and tumor growth in GBM. Thus, Nrf2 is critical in cancer cell pathobiology and targeting Nrf2 activity in cancer, particularly in cancer with Keap1 mutations, may be a promising clinical strategy to inhibit tumor growth.
$\mathrm{Ki}-67$, as a widely used proliferation marker, is associated with cell cycle activity and is expressed at various levels during the G1, S, G2, and M phases, with the exception of the G0 phase (23). Thus, we could perform immunohistochemical staining to detect $\mathrm{Ki}-67$ during the peak level in mitosis. The protein level of Ki-67 shows a good correlation with the growth fraction in several model systems, and its expression has therefore been investigated in various types of human cancer, including glioma (33). Herein, our immunohistochemical results of tumor tissues indicated inhibition of Nrf2 repressed $\mathrm{Ki}-67$ immunoreactivity, indicating that the effects of Nrf2 on tumor growth could be attributed to Nrf2-mediated active and rapid tumor cell division. Regarding the molecular mechanisms, tumor cell proliferation might be dually regulated by epidermal growth factor receptor (EGFR) signaling and the Nrf2 repressor protein Keap1 (14).

Tumor growth is considered the consequence of balancing tumor cell proliferation and spontaneous cell death (34). Apoptosis is an important cell death form and a critical process in cancer cells. It is deregulated resulting in tumorigenesis and drug resistance. In general, the rate of apoptosis in malignant gliomas is very low, and induction of apoptosis may be an important therapeutic strategy for malignant glioma patients. A study recently demonstrated Nrf2 upregulated anti-apoptotic protein $\mathrm{Bcl}-2$, leading to a decrease in Bax, cytochrome $c$ release from mitochondria, activation of caspases, decreased DNA fragmentation and preventing etoposide-induced apoptotic cell death (35). The present study indicated inhibition of Nrf2 upregulated caspase-3 expression, which confirmed the Nrf2-mediated anti-apoptotic effect in cancer. As a result, increased apoptosis by inhibiting Nrf2 expression cannot be excluded from the cause of tumor growth.

It is well established that the growth, invasion and metastasis of most solid malignant tumors are angiogenesis- 
dependent. Kim et al (16) performed a study in colon cancer cells using stable RNAi-mediated knockdown of Nrf2. Their study showed retarded tumor growth, diminished vascular formation, lower VEGF expression and suppressed angiogenesis, and these may all be contributed to Nrf2-mediated HIF- $1 \alpha$ inhibition through the adaptive reduction in mitochondrial $\mathrm{O}_{2}$ consumption. Another study demonstrated that carbon monoxide produced by HO-1 increased HIF-1 $\alpha$ stability and enhanced VEGF expression (36). As one of the downstream genes of Nrf2, HO-1 plays a role in the control of mitochondrial function. As a result, suppressed HO-1 activity by the inhibition of Nrf2 may contribute to reduced mitochondrial function and consequent HIF-1 $\alpha$ defect (16). These may explain our observation of decreased MVD value in the Si-Nrf2 group xenograft tumors.

The current study demonstrated the overexpression of $\mathrm{Nrf} 2$ in GBM samples and correlated it with the clinical prognosis. We further showed the role of Nrf2 in cell proliferation and tumor growth in vitro and in vivo. Our findings support the potential use of Nrf2 siRNA gene therapy for GBM patients. However, the study needs to be repeated in more cell lines and in vivo. In addition, the underlying molecular mechanisms of Nrf2 in cancer remain to be determined by further studies.

\section{Acknowledgements}

The present study was supported partly by the Natural Science Foundation of China (81271377 to L.Z. and 81070974 to H.W.). We are grateful to Bo Yu, Juehua Zhu, Yanwen Yao and Genbao Feng for their technical assistance.

\section{References}

1. Wen PY and Kesari S: Malignant gliomas in adults. N Engl J Med 359: 492-507, 2008.

2. Sullivan PR: Brain tumors. N Engl J Med 344: 1478; author reply $1479,2001$.

3. Chen J, McKay RM and Parada LF: Malignant glioma: lessons from genomics, mouse models, and stem cells. Cell 149: 36-47, 2012.

4. Lau A, Villeneuve NF, Sun Z, Wong PK and Zhang DD: Dual roles of Nrf2 in cancer. Pharmacol Res 58: 262-270, 2008.

5. Dinkova-Kostova AT, Liby KT, Stephenson KK, et al: Extremely potent triterpenoid inducers of the phase 2 response: correlations of protection against oxidant and inflammatory stress. Proc Natl Acad Sci USA 102: 4584-4589, 2005.

6. Kwak MK, Itoh K, Yamamoto M, Sutter TR and Kensler TW: Role of transcription factor Nrf2 in the induction of hepatic phase 2 and antioxidative enzymes in vivo by the cancer chemoprotective agent, 3H-1, 2-dimethiole-3-thione. Mol Med 7: 135-145, 2001.

7. Shih AY, Li P and Murphy TH: A small-molecule-inducible Nrf2mediated antioxidant response provides effective prophylaxis against cerebral ischemia in vivo. J Neurosci 25: 10321-10335, 2005.

8. Yan W, Wang HD, Feng XM, Ding YS, Jin W and Tang K: The expression of NF-E2-related factor 2 in the rat brain after traumatic brain injury. J Trauma 66: 1431-1435, 2009.

9. Wang J, Fields J, Zhao C, et al: Role of Nrf2 in protection against intracerebral hemorrhage injury in mice. Free Radic Biol Med 43: 408-414, 2007.

10. Hayes JD and McMahon M: NRF2 and KEAP1 mutations: permanent activation of an adaptive response in cancer. Trends Biochem Sci 34: 176-188, 2009.

11. Hayes JD and McMahon M: The double-edged sword of Nrf2: subversion of redox homeostasis during the evolution of cancer. Mol Cell 21: 732-734, 2006.

12. Kensler TW and Wakabayashi N: Nrf2: friend or foe for chemoprevention? Carcinogenesis 31: 90-99, 2010.
13. Homma S, Ishii Y, Morishima Y, et al: Nrf2 enhances cell proliferation and resistance to anticancer drugs in human lung cancer. Clin Cancer Res 15: 3423-3432, 2009.

14. Yamadori T, Ishii Y, Homma S, et al: Molecular mechanisms for the regulation of Nrf2-mediated cell proliferation in non-smallcell lung cancers. Oncogene 45: 4768-4777, 2012.

15. Lister A, Nedjadi T, Kitteringham NR, et al: Nrf2 is overexpressed in pancreatic cancer: implications for cell proliferation and therapy. Mol Cancer 10: 37, 2011.

16. Kim TH, Hur EG, Kang SJ, et al: NRF2 blockade suppresses colon tumor angiogenesis by inhibiting hypoxia-induced activation of HIF-1 $\alpha$. Cancer Res 71: 2260-2275, 2011.

17. Pan H, Wang H, Zhu L, Mao L, Qiao L and Su X: The role of Nrf2 in migration and invasion of human glioma cell U251. World Neurosurg: Nov 7, 2011 (Epub ahead of print).

18. Rousseau A, Mokhtari K and Duyckaerts C: The 2007 WHO classification of tumors of the central nervous system - what has changed? Curr Opin Neurol 21: 720-727, 2008.

19. Bustin SA, Benes V, Garson JA, et al: The MIQE guidelines: minimum information for publication of quantitative real-time PCR experiments. Clin Chem 55: 611-622, 2009.

20. Hu Z, Lin D, Yuan J, et al: Overexpression of osteopontin is associated with more aggressive phenotypes in human non-small cell lung cancer. Clin Cancer Res 11: 4646-4652, 2005.

21. Weidner N, Semple JP, Welch WR and Folkman J: Tumor angiogenesis and metastasis--correlation in invasive breast carcinoma. N Engl J Med 324: 1-8, 1991.

22. Wang M, Tang J, Liu S, Yoshida D and Teramoto A: Expression of cathepsin B and microvascular density increases with higher grade of astrocytomas. J Neurooncol 71: 3-7, 2005.

23. Yerushalmi R, Woods R, Ravdin PM, Hayes MM and Gelmon KA: Ki67 in breast cancer: prognostic and predictive potential. Lancet Oncol 11: 174-183, 2010.

24. Moi P, Chan K, Asunis I, Cao A and Kan YW: Isolation of NF-E2-related factor 2 (Nrf2), a NF-E2-like basic leucine zipper transcriptional activator that binds to the tandem NF-E2/AP1 repeat of the beta-globin locus control region. Proc Natl Acad Sci USA 91: 9926-9930, 1994.

25. Padmanabhan B, Tong KI, Ohta T, et al: Structural basis for defects of Keap1 activity provoked by its point mutations in lung cancer. Mol Cell 21: 689-700, 2006.

26. Singh A, Misra V, Thimmulappa RK, et al: Dysfunctional KEAP1-NRF2 interaction in non-small-cell lung cancer. PLoS Med 3: e420, 2006.

27. Shibata T, Kokubu A, Gotoh M, et al: Genetic alteration of Keap1 confers constitutive Nrf2 activation and resistance to chemotherapy in gallbladder cancer. Gastroenterology 135: 1358-1368, 2008.

28. Nioi P and Nguyen T: A mutation of Keap1 found in breast cancer impairs its ability to repress Nrf2 activity. Biochem Biophys Res Commun 362: 816-821, 2007.

29. Shibata T, Ohta T, Tong KI, et al: Cancer related mutations in NRF2 impair its recognition by Keap1-Cul3 E3 ligase and promote malignancy. Proc Natl Acad Sci USA 105: 13568-13573, 2008.

30. Konstantinopoulos PA, Spentzos D, Fountzilas E, et al: Keap1 mutations and nrf2 pathway activation in epithelial ovarian cancer. Cancer Res 71: 5081-5089, 2011.

31. Merikallio H, Paakko P, Kinnula VL, Harju T and Soini Y: Nuclear factor erythroid-derived 2-like 2 (Nrf2) and DJ1 are prognostic factors in lung cancer. Hum Pathol 43: 577-584, 2011.

32. Singh A, Boldin-Adamsky S, Thimmulappa RK, et al: RNAimediated silencing of nuclear factor erythroid-2-related factor 2 gene expression in non-small cell lung cancer inhibits tumor growth and increases efficacy of chemotherapy. Cancer Res 68: 7975-7984, 2008.

33. Wiesner FG, Magener A, Fasching PA, et al: Ki-67 as a prognostic molecular marker in routine clinical use in breast cancer patients. Breast 18: 135-141, 2009.

34. Thompson CB: Apoptosis in the pathogenesis and treatment of disease. Science 267: 1456-1462, 1995.

35. Niture SK and Jaiswal AK: Nrf2 up-regulates anti-apoptotic protein Bcl-2 and prevents cellular apoptosis. J Biol Chem 287: 9873-9886, 2012.

36. Choi YK, Kim CK, Lee H, et al: Carbon monoxide promotes VEGF expression by increasing HIF-1 $\alpha$ protein level via two distinct mechanisms, translational activation and stabilization of HIF-1 $\alpha$ protein. J Biol Chem 285: 32116-32125, 2010. 